\title{
Inteligência artificial aplicada ao direito e o direito da inteligência artificial ${ }^{1}$
}

\author{
Artificial intelligence applied to the law and the law of artificial \\ intelligence
}

\author{
Juliano Souza de Albuquerque Maranhão \\ Universidade de São Paulo (São Paulo, SP, Brasil) \\ ORCID: https://orcid.org/0000-0002-2705-7440 \\ E-mail: julianomaranhao@usp.br
}

\begin{abstract}
Juliana Abrusio Florêncio ${ }^{3}$
Universidade Presbiteriana Mackenzie (São Paulo, SP, Brasil)

ORCID: https://orcid.org/0000-0002-3745-0748

E-mail: juliana@opiceblum.com.br
\end{abstract}

Marco Almada ${ }^{4}$

Instituto Universitário Europeu (Fiesole, Florença, Itália) ORCID: https://orcid.org/0000-0002-0127-6549

E-mail: Marco.Almada@eui.eu

\section{Resuino}

O desenvolvimento de tecnologias de inteligência artificial é relevante para o Direito por duas razões distintas, mas relacionadas. De um lado, a adoção de tecnologias inteligentes em aplicações no setor público e privado, em especial em contextos de tomada de decisão automatizada, atrai demandas regulatórias para o surgimento de um Direito da inteligência artificial; de outro, o próprio Direito surge

\footnotetext{
${ }^{1}$ MARANHÃO, Juliano Souza de Albuquerque; FLORÊNCIO, Juliana Abrusio; ALMADA, Marco. Inteligência artificial aplicada ao direito e o direito da inteligência artificial. Suprema: revista de estudos constitucionais, Brasília, v. 1, n. 1, p. 154-180, jan./ jun. 2021.

${ }^{2}$ Professor Associado do Departamento de Filosofia e Teoria Geral do Direito da USP. Membro do Comitê Diretor da Associação Internacional de Inteligência Artificial e Direito. Diretor do Lawgorithm. Currículo Lattes: https://lattes.cnpq. $\mathrm{br} / 3498148304153540$.

${ }^{3}$ Doutora em Direito pela PUC-SP. Professora da Universidade Presbiteriana Mackenzie. Pesquisadora do Lawgorithm. Currículo Lattes: https://lattes.cnpq.br/1847187277633756.

${ }^{4}$ Doutorando em Direito no Instituto Universitário Europeu, com bolsa da Fundación Carolina. Mestre em Engenharia Elétrica pela Unicamp. Bacharel em Direito pela USP. Pesquisador do Lawgorithm. Currículo Lattes: https://lattes.cnpq. br/5522088247944723.
} 
como um domínio para a aplicação da inteligência artificial, que tem o potencial de impactar as profissões jurídicas. O presente artigo examina o estado atual da literatura a respeito desses dois temas e a interconexão entre essas duas perspectivas a respeito do fenômeno da inteligência artificial. Ao examinar possíveis soluções para os problemas regulatórios e de aplicação, o artigo propõe temas para a pesquisa na interface entre Direito e tecnologia e ressalta a importância de pesquisas que integrem estas perspectivas, envolvendo pesquisadores de diversas áreas em torno das questões jurídicas.

\section{Palavras-chave}

Proteção de dados; Inteligência artificial; Decisões automatizadas; Automação jurídica; Regulação da inteligência artificial.

\section{Sumário}

1. Introdução. 2. A regulação de sistemas inteligentes. 2.1. Opacidade de sistemas computacionais. 2.2. Princípios éticos e iniciativas regulatórias. 3. A aplicação da inteligência artificial ao Direito. 3.1. Sistemas baseados em conhecimento jurídico. 3.2. Aplicações jurídicas do aprendizado de máquina. 3.3. Inteligência artificial no Direito brasileiro. 4. Perspectivas da inteligência artificial aplicada ao Direito. 4.1. Abordagens híbridas e o problema da explicação das decisões. 4.2. Direito computável. 5. Conclusão.

\section{Abstract}

The development of artificial intelligence technologies is legally relevant for two distinct, but related, reasons. On the one hand, the adoption of intelligent technologies in public and private sector applications, especially in automated decision-making contexts, inspires regulatory demands for the creation of artificial intelligence law; on the other hand, the law itself appears as a domain for applying artificial intelligence, with the potential for impacting the legal professions. This paper examines the current state of the literature on both topics, as well as the interconnections between those two perspectives on artificial intelligence. By examining possible solutions for the regulatory and application issues, this paper proposes themes for research in the interface between law and technology, thus highlighting the importance of interdisciplinary research centred in such legal questions. 


\section{Keywords}

Data protection; Artificial intelligence; Automated decision-making; Legal automation; Artificial intelligence regulation.

\section{Contents}

1. Introduction. 2. The regulation of intelligent systems. 2.1. Opaque computational systems. 2.2. Ethical principles and regulatory initiatives. 3. Applying artificial intelligence to the law. 3.1. Legal knowledge systems. 3.2. Legal applications of machine learning. 3.3. Artificial intelligence in Brazilian law. 4. Perspectives for artificial intelligence and law. 4.1. Hybrid approaches and the problem of explaining decisions. 4.2. Computable law. 5. Conclusion.

\section{Introdução}

A inteligência artificial (IA) é um tema cada vez mais presente nos debates jurídicos brasileiros. ${ }^{5}$ Tal popularização não é meramente um exercício especulativo, inspirado por séries de ficção científica como Black Mirror, mas reflete os desenvolvimentos tecnológicos dos últimos anos. Em alguns casos, o engajamento com sistemas de inteligência artificial é mais diretamente visível - por exemplo, na proliferação de chatbots e assistentes pessoais em celulares -, enquanto em outros a tecnologia opera nos bastidores, como ocorre no crescente uso de tecnologias de automação pelo judiciário brasileiro. Mas, de formas visíveis ou invisíveis, a inteligência artificial já afeta as vidas de milhões de brasileiros, suscitando perguntas a respeito de como o Direito deve lidar com essas novas tecnologias.

No presente artigo, analisamos a relevância da inteligência artificial para o Direito a partir de duas perspectivas distintas, mas complementares.

A primeira perspectiva parte da crescente adoção de sistemas inteligentes em várias aplicações, tanto na tomada de decisões nos setores públicos e privados quanto na construção de sistemas voltados ao consumidor e assistentes pessoais para as mais diversas tarefas cotidianas. Tal proliferação de inteligências artificiais significa seu envolvimento ubíquo em diversas relações sociais e econômicas tuteladas pelo

\footnotetext{
${ }^{5}$ Uma pesquisa realizada em 25 de março de 2021 no Google Acadêmico (https://scholar.google.com) com as palavras-chave "inteligência artificial"+direito retornou cerca de 24 mil artigos, dos quais aproximadamente metade foi publicada a partir de 2017. O número é consideravelmente maior para a mesma pesquisa realizada em língua inglesa: "artificial intelligence" + law retorna pouco menos de um milhão de publicações, das quais 110 mil foram realizadas nos últimos quatro anos.
} 
Direito. Neste cenário, podemos falar de um Direito da Inteligência Artificial, ou seja, da disciplina jurídica dos agentes digitais e das implicações de seu envolvimento em relações jurídicas e conflitos delas decorrentes.

A segunda decorre do fato de a inteligência artificial ser não apenas um objeto externo sujeito à disciplina jurídica, mas também uma ferramenta cada vez mais utilizada por operadores do Direito. Apesar de o emprego de aprendizado de máquina ter apresentado resultados extremamente úteis para advogados e para tribunais, especialmente com análise preditiva, arguiremos aqui que as correlações empíricas utilizadas por esses sistemas enfrentam limitações em sua capacidade explicativa, o que compromete aplicações no domínio jurídico, no qual há exigência de justificação normativa das decisões. Acreditamos que a próxima geração de inteligência artificial aplicada ao Direito deverá incorporar modelos de representação de conhecimento jurídico às ferramentas de aprendizado de máquina.

De forma a lastrear e contextualizar as afirmações apresentadas acima, o presente artigo efetua uma revisão não sistemática da literatura jurídica a respeito da inteligência artificial. Essa revisão tem enfoque na apresentação do estado da arte do debate internacional e é articulada em torno de dois eixos: os debates regulatórios em torno da inteligência artificial e os avanços e limites da aplicação de técnicas de inteligência artificial em domínios jurídicos. Tal mapa, por sua vez, serve de base para a contextualização do recente crescimento nas publicações e ferramentas desenvolvidas no Brasil, em face dos dados empíricos existentes a respeito da adoção de IA no país, bem como para a análise das futuras direções de desenvolvimento das aplicações de inteligência artificial ao Direito e dos problemas legais e regulatórios associados ao desenvolvimento dessas tecnologias.

$\mathrm{Na}$ próxima seção, apresentaremos os principais desafios envolvidos na regulação da inteligência artificial, cujas consequências jurídicas serão exploradas na Seção 3. Essas duas perspectivas estão relacionadas entre si, já que a disciplina jurídica da inteligência artificial influenciará os sistemas construídos para finalidades jurídicas, bem como a tecnologia pode tornar viáveis novas abordagens regulatórias. A Seção 4 explora algumas possibilidades futuras de integração entre o Direito da inteligência artificial e a inteligência artificial aplicada ao Direito. Portanto, a atuação interdisciplinar entre os profissionais do Direito, da Engenharia e da Computação pode ser benéfica para todas essas áreas, contribuindo para o funcionamento do sistema jurídico e para que as aplicações de inteligência artificial não lesionem, ou até mesmo promovam, os direitos e interesses protegidos pelo Direito. 


\section{A regulação de sistemas inteligentes}

Um algoritmo é qualquer procedimento de computador bem definido que possua algum valor agregado na qualidade de suas entradas (input), gerando outros valores na saída (output), de forma que pode ser considerado uma ferramenta para resolver um problema (CORMEN et al., 2009, p. 5-6). Os algoritmos são empregados em programas de computador por diversas organizações para a tomada de decisões e alocação de recursos a partir de grandes conjuntos de dados. Entre esses algoritmos, adquiriram particular relevo nos últimos anos os chamados algoritmos de inteligência artificial, que utilizam técnicas específicas para a construção de sistemas capazes de agir racionalmente diante de situações específicas (RUSSELL; NORVIG, 2010, p. 4-5). ${ }^{6}$

Combinando cálculo, processamento e raciocínio, os sistemas que implementam algoritmos de inteligência artificial podem ser excepcionalmente complexos, codificando milhares de variáveis em milhões de pontos de dados (CAPLAN et al., 2018). Desse modo, decisões importantes sobre a vida das pessoas, como processos seletivos de empresas e concessão de crédito, são cada vez mais ocasionadas por sistemas ditos inteligentes. Surge, portanto, a preocupação a respeito de como programas de computador que utilizam técnicas de inteligência artificial podem limitar oportunidades e principalmente colocar em risco direitos fundamentais dos cidadãos.

Entre os empregos de sistemas de inteligência artificial, um dos principais focos de atenção para o Direito está na coleta e tratamento de dados pessoais para perfilamento. ${ }^{7}$ Determinar o perfil do indivíduo pode valer muito a anunciantes, a seguradoras, e ao próprio Estado. A China, por exemplo, tem utilizado uma combinação de vigilância por meio de inteligência artificial com uso de enorme quantidade de dados pessoais para monitorar a vida e o comportamento das pessoas em detalhes minuciosos (GOMES, 2017, p. 50-51; LARSON, 2018; ONG, 2011, p. 172-179). Nesse sentido, surge na literatura acadêmica e na sociedade civil uma preocupação a

\footnotetext{
${ }^{6}$ Nem todo algoritmo é uma manifestação de inteligência artificial. Por exemplo, o método que se aprende no Ensino Fundamental para a divisão de dois números inteiros é um algoritmo, que recebe como inputs o divisor e o dividendo e fornece como outputs o resultado da divisão e o resto (que pode ser zero), mas uma máquina que apenas execute esse algoritmo dificilmente será considerada inteligente. Contudo, boa parte dos métodos que hoje conhecemos para a produção de sistemas que manifestem inteligência artificial - ainda que em um sentido amplo de inteligência que não equivale à inteligência humana (BRYSON; THEODOROU, 2019) - envolvem algoritmos sofisticados que são implementados e executados em computadores digitais.

${ }^{7}$ Segundo o artigo 4(4) do Regulamento Geral de Proteção de Dados da União Europeia (GDPR, na sigla em inglês), "perfilização" é qualquer forma de tratamento automatizado de dados pessoais que consista em utilizar esses dados pessoais para avaliar certos aspectos pessoais de uma pessoa singular, nomeadamente para analisar ou prever aspectos relacionados com desempenho profissional, situação econômica, saúde, preferências pessoais, interesses, fiabilidade, comportamento, localização ou deslocações. No Brasil, a Lei Geral de Proteção de Dados Pessoais (LGPD) não define o que entende por perfis, mas faz uso do conceito em dois dispositivos: no artigo 12 (dados anonimizados) e no artigo 20 (decisões automatizadas).
} 
respeito dos limites da chamada governança algorítmica (MACHADO, 2018) e de seus impactos no Estado de Direito (BAYAMLIOĞLU; LEENES, 2018).

De um ponto de vista jurídico, essa preocupação conecta-se com o desafio de como contestar e revisar decisões lastreadas em inteligência artificial, principalmente quando baseadas em técnicas computacionais opacas (black box). ${ }^{8}$ A contestabilidade e a possibilidade de revisão pressupõem inteligibilidade, em termos humanos, ou seja, a existência de um conjunto de critérios determinantes que fundamentem certa decisão. A existência de tais critérios, por sua vez, é essencial para que indivíduos e grupos possam identificar eventuais efeitos adversos do uso da inteligência artificial e buscar os remédios cabíveis, seja pela via judicial ou por outros caminhos lícitos. ${ }^{9}$ Passaremos, portanto, ao exame das formas como sistemas de inteligência artificial podem ser opacos aos observadores externos.

\subsection{Opacidade de sistemas computacionais}

A opacidade de sistemas de aprendizado de máquina é uma das maiores fontes de atenção e preocupação na atualidade, principalmente no que diz respeito à possibilidade de contestação, mas também em relação ao risco de incorporação de vieses que resultem em construção de perfis ou tomadas de decisão discriminatórias, ou ainda da possibilidade de tomadas de decisão que ignorem valores humanos ou desrespeitem direitos fundamentais e a dignidade humana. ${ }^{10}$

Três fatores contribuem, em variados graus, para que um sistema seja opaco: a complexidade dos modelos matemáticos envolvidos, a dificuldade de entender as operações envolvidas no processamento de dados em larga escala e a falta de clareza no contexto institucional de uso destes sistemas (BURRELL, 2016). A lógica de funcionamento de sistemas de inteligência artificial envolve modelos computacionais de variados graus de complexidade, que servem de ponto de partida para o desenvolvimento de programas de computador para realizar uma determinada tarefa (LEHR; OHM, 2017). Entender esses sistemas é, portanto, uma tarefa que exige conhecimento especializado (BURRELL, 2016, p. 4), mas até mesmo especialistas podem ter dificuldades para compreender todas as variáveis e fatores envolvidos no

\footnotetext{
${ }^{8}$ Jatinder Singh et al. (2016, p.7) destacam a dificuldade em manter o controle sobre sistemas opacos.

${ }^{9}$ Para um tratamento do direito à contestação de decisões judiciais no âmbito da automação de decisões, ver Claudio Sarra (2020).

${ }^{10} \mathrm{O}$ tema da discriminação algorítmica é, por si só, objeto de uma vasta literatura acadêmica, cuja síntese foge ao escopo deste artigo. Recente pesquisa efetuada por Letícia Simões-Gomes, Enrico Roberto e Jônatas Mendonça (2020) mostra que há uma escassez de produção sobre o tema nas línguas portuguesa e espanhola, que se manifesta em vagueza conceitual e produção primariamente ensaística no cunho das humanidades. Há, contudo, um incipiente corpo de estudos lusófonos que abordam o tema de forma mais sistemática, como a tipologia proposta por Laura Schertel Mendes e Marcela Mattiuzzo (2019).
} 
tratamento de dados em escala de big data (BURRELL, 2016, p. 4-5), em especial quando se leva em conta a presença de segredos industriais e comerciais, ou mesmo de Estado, que podem dificultar o acesso aos algoritmos por trás de sistemas computacionais ou das ferramentas usadas para sua implementação (BURRELL, 2016, p. 3-4).

A presença de um ou mais desses fatores pode dificultar a identificação de lesões ou ameaças a direitos e interesses, sejam eles individuais ou coletivos, que surjam em decorrência do uso da inteligência artificial. Essa dificuldade pode, inclusive, servir de instrumento para que empresas e outros controladores de dados, ao manter suas operações opacas, evitem ou confundam os esforços regulatórios (BURRELL, 2016, p. 3-4; PASQUALE, 2015, p. 2). Como alerta o jurista americano Frank Pasquale (2015, p. 3), as diversas formas de opacidade que cercam os algoritmos fazem com que estejamos no escuro em relação a decisões cruciais.

Para Pasquale, deve haver menor esforço de concentração em tentar controlar a coleta de dados, e mais esforço em regular o uso desses dados - como as empresas e os governos estão realmente implantando regras para tomar decisões, com emprego de inteligências artificiais (PASQUALE, 2015, p. 141). É em relação a esses riscos que se direcionam esforços não só jurídicos, como também de incorporação de critérios éticos no desenvolvimento de sistemas.

\subsection{Princípios éticos e iniciativas regulatórias}

Nos últimos anos, diversos órgãos governamentais, associações de pesquisa e organizações privadas produziram documentos em resposta às preocupações acerca do emprego de sistemas de inteligência artificial, propondo parâmetros éticos para o desenvolvimento e aplicação de sistemas de IA. De acordo com um estudo de Anna Jobin, Marcello Ienca e Effy Vayena (2019), há ao menos 84 documentos contendo princípios ou diretrizes para a inteligência artificial. Esses documentos são publicados por governos, organizações internacionais, empresas e organizações do terceiro setor, que por vezes adotam princípios distintos uns dos outros ou dão ênfases diferentes aos princípios escolhidos.

Nos diversos documentos, há alguma convergência em torno dos princípios de transparência (deve estar claro para o usuário que ele interage com um sistema artificial), explicabilidade (divulgação de informações ao interessado que permitam ao usuário entender os critérios para tomada de decisão), não discriminação (evitar que os sistemas incorporem vieses que possam ofender direitos fundamentais), não maleficência (sistemas de IA não podem prejudicar humanos), responsabilidade e privacidade/proteção de dados, muito embora haja divergências sobre o significado e a forma de implementação destes princípios (JOBIN; IENCA; VAYENA, 2019). 
Um dos atores mais ativos no campo de propostas éticas para a inteligência artificial é a União Europeia, que atua por meio de suas diversas instâncias, articuladas por um plano coordenado (COMISSÃO EUROPEIA, 2018). Iniciativas nesse sentido podem ser vistas na atuação da autoridade de proteção de dados da União Europeia, ${ }^{11}$ do Parlamento Europeu, ${ }^{12}$ e da Comissão Europeia. ${ }^{13}$ Já no Brasil, o Ministério da Ciência, Tecnologia e Inovação abriu, no final de 2019, uma consulta pública a respeito da elaboração de uma Estratégia Nacional de Inteligência Artificial, que tem entre seus pilares o estabelecimento de diretrizes éticas para sistemas inteligentes, ${ }^{14}$ ao passo que o Conselho Nacional de Justiça (CNJ) emitiu a Resolução 332/2020, estabelecendo requisitos éticos, de transparência e de governança que devem ser observados no uso de sistemas inteligentes em contextos judiciais.

O estabelecimento de princípios éticos para guiar o desenvolvimento da inteligência artificial é necessário para conduzir a aplicação da inteligência artificial para finalidades socialmente positivas, mas envolve alguns riscos. Uma primeira dificuldade está relacionada ao nível adequado de uso da inteligência artificial. De um lado, há a possibilidade de sobreutilização, com o emprego de sistemas inteligentes em situações que podem trazer impacto negativo aos seres humanos; de outro, o temor desta possibilidade pode levar à subutilização da inteligência artificial, impedindo a sociedade de aproveitar os benefícios que podem ser extraídos dessas tecnologias (FLORIDI et al., 2018).

\footnotetext{
${ }^{11}$ Em dezembro de 2015, o European Data Protection Supervisor criou um Ethics Advisory Group voltado às dimensões éticas da proteção de dados no atual contexto digital. Em 2018, este grupo produziu um relatório em que sustenta que a nova ética digital tem como fundação o direito fundamental à privacidade e à proteção de dados pessoais, entendendo crucial essa observância ética para a proteção da dignidade humana, que é o cerne da Carta de Direitos Fundamentais da União Europeia (BURGESS et al., 2018).

${ }^{12}$ Entre outros, ver Koene et al. (2019). Este relatório desenvolve e propõe opções de regulações para a governança da transparência algorítmica e de sua responsabilização, a partir da análise dos aspectos sociais, técnicos e regulatórios.

${ }^{13}$ Em abril de 2021, a Comissão Europeia introduziu uma proposta de regulação voltada a disciplinar o uso de tecnologias de inteligência artificial (COMISSÃO EUROPEIA, 2021). Esta proposta legislativa, que se baseia nas competências da União Europeia para a proteção de dados pessoais e do mercado comum, separa os sistemas inteligentes em três categorias: aplicações proibidas (art. $5^{\circ}$ ), cujo uso é de antemão descrito como incompatível com o ordenamento jurídico da União Europeia; aplicações de alto risco, descritas no art. $6^{\circ}$; e as demais aplicações, nas quais a presença de inteligência artificial não atrai deveres jurídicos adicionais, exceto pelo dever de transparência que o art. 52 impõe a certas aplicações consideradas de baixo risco. Para as aplicações tidas como de alto risco, a proposta introduz uma série de deveres procedimentais: a necessidade de acompanhamento contínuo dos riscos ligados ao uso de um sistema inteligente (art. 9º e da precisão, robustez e segurança da informação deste sistema (art. 15), de controle a respeito dos dados usados para alimentar o sistema (art. 10), de manutenção de documentação técnica e registros da operação de sistema (art. 11-12), de prestação transparente de informações para os usuários do sistema (art. 13) e da adoção de mecanismos para supervisão humana da operação destes sistemas (art. 14). No momento da escrita do artigo, a proposta da Comissão Europeia ainda deve passar por todo o processo legislativo da União Europeia, mas a formulação inicial da proposta regulatória sinaliza uma ênfase na transparência e adoção de mecanismos técnicos de controle de sistemas de alto risco, em adição aos deveres gerais impostos pelo ordenamento jurídico da União Europeia em matéria de proteção de dados pessoais.

${ }^{14}$ Para o anúncio da consulta pública, ver https://www.mctic.gov.br/mctic/opencms/salaImprensa/noticias/arquivos/2019/12/ MCTIC_lanca_consulta_publica_para_a_Estrategia_Brasileira_de_Inteligencia_Artificial.html. Até o momento da publicação deste artigo, o Ministério ainda não havia divulgado uma resposta pública ou uma compilação das contribuições. Todavia, a chamada contou com a participação de grande número de cidadãos e organizações da sociedade civil.
} 
Outra dificuldade está no fato de que os princípios são formulados em discussões "top-down" e pretensão de universalidade, o que torna seu conteúdo demasiadamente genérico e abstrato, tornando-os de difícil aplicação. Por um lado, a definição de regras gerais em vez de uma regulação rígida é favorável ao desenvolvimento tecnológico e permite que a sociedade se familiarize com os sistemas inteligentes antes de decidir como eles devem ser regulados. Por outro, ela pode contribuir para o que a literatura sobre regulação de inteligência artificial chama de ethics-washing: o uso de princípios vagos como um substituto para regras que efetivamente protegeriam os direitos e interesses individuais e coletivos que poderiam ser afetados pelo uso de sistemas inteligentes. ${ }^{15}$

Assim, um dos grandes desafios na regulação da inteligência artificial consiste em identificar não só os princípios cabíveis, mas os momentos em que esses princípios devem ser implementados por regras jurídicas, bem como os instrumentos legais mais adequados para esta regulação. ${ }^{16}$ Entendemos que o melhor caminho para discussões éticas que pretendam aplicabilidade seja por meio da análise "bottom-up", buscando equilíbrio reflexivo entre princípios gerais e casos concretos em setores específicos. ${ }^{17}$ Ou seja, trata-se de discutir não os princípios universais da ética computacional ou algorítmica, mas de desenhar princípios específicos para diferentes setores de aplicação: ética algorítmica no campo da medicina, no campo jurídico, no âmbito comercial, etc. Refletindo as prioridades existentes em cada domínio de aplicação, será possível construir sistemas inteligentes que sejam centrados nas demandas humanas (BRYSON; THEODOROU, 2019; COMISSÃO EUROPEIA, 2018).

O equilíbrio ideal dependerá não só das capacidades tecnológicas dos sistemas inteligentes, mas também das peculiaridades de cada domínio de aplicação e das exigências da lei. Nesse sentido, é salutar o desenvolvimento de uma literatura acadêmica brasileira sobre o tema que, em diálogo com o estado da arte da pesquisa global, busca enfrentar problemas no contexto brasileiro e dentro das condições do ordenamento pátrio, sem, contudo, perder de vista que muitos desses desafios também se fazem presentes em outros países. ${ }^{18}$

\footnotetext{
${ }^{15}$ Para um mapeamento do fenômeno de ethics-washing, ver Ben Wagner (2018). Elettra Bietti (2020) oferece uma visão alternativa, que busca encontrar um papel para a ética da tecnologia enquanto evita sua captura por interesses de evasão regulatória.

${ }^{16}$ Sobre a busca pelo equilíbrio adequado, ver Juliano Maranhão e Diogo Coutinho (2019). Para um exemplo concreto dos riscos envolvidos na regulação de tecnologia, ver Gus Swanda (2016).

${ }^{17}$ Como discutido por, entre outros, Juliano Maranhão e Marco Almada (2021).

${ }^{18}$ Uma primeira coletânea nacional a respeito do tema foi organizada por Ana Frazão e Caitlin Mulholland (2019). No tratamento sistêmico das implicações da inteligência artificial para o Direito, Juliana Abrusio (2020) analisa as implicações da "cultura do algoritmo" para o regime de proteção de dados no Brasil e no mundo - tema também enfrentado por, entre outros, Danilo Doneda et al. (2018). A respeito das implicações destas considerações sistemáticas para temas jurídicos, ver, entre outros, Maranhão e Almada (2021) no campo da saúde, e Luiz Fux; Henrique Ávila; e Trícia Navarro Xavier Cabral (2021) a respeito do impacto da tecnologia no acesso à justiça, bem como diversos dos capítulos da já mencionada obra de Frazão e Mulholland (2019).
} 
No caso da inteligência artificial aplicada ao Direito, por exemplo, um desafio compartilhado e particularmente forte é aquele posto pelo problema da "black box" discutido acima, já que as decisões judiciais estão sujeitas a uma série de requisitos que ultrapassam a mera acurácia preditiva, como a necessidade de que o conteúdo da decisão possa ser justificado racionalmente. A seguir, exploraremos em mais detalhes os usos jurídicos da inteligência artificial e o estado da arte em relação ao problema de tornar explicáveis as decisões desses sistemas.

\section{A aplicação da inteligência artificial ao Direito}

Sistemas de inteligência artificial são usados, como vimos acima, para automatizar várias tarefas cuja realização exigiria a inteligência humana. Entre essas tarefas, é particularmente relevante o uso de sistemas inteligentes para tentar prever o comportamento de indivíduos - o que engloba objetivos diversos, como a identificação das preferências de consumo de alguém e a determinação da probabilidade de inadimplência em um financiamento ${ }^{19}-$, bem como o uso de dados para a tomada de decisão sem a participação humana.

Esses modelos, como discutidos acima, podem ser opacos ao entendimento humano, o que pode ser resultado de três fontes principais: a complexidade dos modelos matemáticos envolvidos, a dificuldade de entender as operações envolvidas no processamento de dados em larga escala e a falta de clareza no contexto institucional de uso desses sistemas. Como as atividades jurídicas - seja na esfera judicial, seja na esfera administrativa, seja em outros métodos de solução de controvérsias - estão em geral conectadas a situações que podem produzir impactos significativos para pessoas físicas e jurídicas, o ordenamento jurídico impõe uma série de restrições que devem ser seguidas para o uso lícito da inteligência artificial como ferramenta de apoio ou de automação.

Uma primeira fonte dessas restrições é o regramento jurídico da privacidade e da proteção de dados pessoais: os princípios éticos já mencionados, bem como as leis inspiradas por eles, também se aplicam aos usos jurídicos da inteligência artificial. Em particular, a Lei Geral de Proteção de Dados Pessoais (LGPD), Lei 13.709/2018, que entrou em vigor em agosto de 2021, traz dispositivos relevantes para aplicações

\footnotetext{
${ }^{19}$ Para uma descrição das possibilidades neste sentido, ver Gomes (2017).
} 
que estejam ligadas a questões jurídicas, como a geração automática de contratos ou a busca automatizada por jurisprudência. ${ }^{20}$

A essas preocupações gerais, lidas à luz da discussão da seção anterior, somam-se as questões específicas do âmbito jurídico. Um exemplo é a exigência de que as decisões judiciais sejam fundamentadas na análise das questões de fato e de direito presentes no processo. ${ }^{21}$ Diante desse requisito, um sistema inteligente que se proponha a automatizar uma decisão judicial - ou, de forma mais realista para a tecnologia atual, a fornecer aportes para um juiz humano - deve ser capaz de fornecer a fundamentação exigida por lei. Porém, a opacidade envolvida no uso e construção dos sistemas inteligentes pode dificultar, ou mesmo tornar inviável, a produção desse tipo de fundamentação.

A LGPD - assim como sua contraparte da União Europeia, o Regulamento Geral de Proteção de Dados (RGPD, ou GDPR na sigla inglesa) - inclui uma forma do chamado direito à explicação. ${ }^{22}$ Segundo a LGPD, em seu artigo 20, $\$ 1^{\circ}$, o controlador de sistemas que tomam decisões amparadas unicamente no tratamento automatizado de dados pessoais deve fornecer informações a respeito dos critérios e procedimentos utilizados para a decisão automatizada. Essa formulação da lei se aproxima do paradigma de sistemas baseados em conhecimento, que fazem uso de representações predefinidas do conhecimento disponível a respeito do problema que pretendem resolver (RUSSELL; NORVIG, 2010, p. 234).

Contudo, muitas das aplicações de inteligência artificial que hoje assumem papel de destaque no imaginário popular seguem outro paradigma técnico: os sistemas de dados. Essa abordagem, nos últimos anos, foi usada para a construção de sistemas de busca por divergências em acórdãos do Supremo Tribunal Federal (OLIVEIRA, 2017), a predição de decisões da Corte Europeia de Direitos Humanos (MEDVEDEVA; VOLS; WIELLING, 2020), e a aplicação de testes de propósito principal na tributação internacional (KUZNIACKI, 2018), entre outras aplicações.

\footnotetext{
${ }^{20}$ A LGPD estabelece, em seu artigo 7o, VI, o exercício regular de direitos em processo judicial, administrativo ou arbitral como uma das hipóteses para o tratamento lícito de dados pessoais. O capítulo IV desta lei (artigos 23 a 32 ) dispõe sobre o tratamento de dados automatizados no poder público, o que abrange o judiciário e o ministério público, bem como as instâncias processuais administrativas.

${ }^{21}$ Vide, e. g., a exigência da fundamentação como elemento essencial da sentença pelo Código de Processo Civil, artigo 489, I.

${ }^{22}$ Para um panorama da discussão a respeito dos limites do direito à explicação na regulação da União Europeia, ver Kaminski (2019). Para uma discussão dos limites de viabilidade da explicação em face das possibilidades tecnológicas, ver Almada (2019).
} 
Sistemas de dados não são construídos a partir de representações predefinidas do problema que pretendem responder. Em vez disso, eles lançam mão do aprendizado de máquina (HAYKIN, 2008, p. 34): antes de serem usados para sua aplicação, esses sistemas são expostos a dados sobre o problema a resolver e, por meio de processos estatísticos de treinamento, ${ }^{23}$ extraem da base de dados padrões e correlações que serão generalizados para resolver problemas futuros.

Esses métodos de predição são empíricos, ou seja, extraem os resultados de uma série de decisões judiciais e as correlacionam com fatores como o tipo de demanda, valor envolvido, e o tribunal em que a demanda é julgada. Como sua estrutura é definida a partir das propriedades detectáveis com base nos dados, os sistemas de aprendizado de máquina não levam em consideração qualquer justificação normativa sobre como deve ser a decisão a partir das características e argumentos do caso.

Além disso, a complexidade matemática dos modelos empregados para extrair as propriedades dos dados torna inviável, mesmo para o observador técnico, o detalhamento minucioso de como esses sistemas operam. Quando essas técnicas são aplicadas em cenários de big data, ${ }^{24}$ a escala das operações envolvidas torna o cenário ainda mais complexo, dificultando a exposição das decisões tomadas pelo sistema em forma compreensível para humanos, com premissas, critérios acessíveis, argumentos e conclusões. ${ }^{25}$

Nesta seção, discutiremos os êxitos e limitações de ambos os paradigmas do aprendizado de máquina. De um lado, os sistemas baseados em conhecimento conseguem representar uma vasta gama de aplicações jurídicas, bem como produzir respostas inteligíveis, mas sua construção e uso exigem um esforço que muitas vezes dificulta aplicações práticas. Já os sistemas de aprendizado de máquina conseguem extrair correlações estatísticas com menos estrutura do que a exigida pelos sistemas que lançam mão de formalizações do conhecimento jurídico, mas suas soluções não são explicáveis em um nível compatível com as exigências jurídicas nem se beneficiam do conhecimento jurídico. Por fim, defenderemos a combinação entre técnicas de conhecimento jurídico e técnicas de aprendizado de máquina, aproveitando os pontos

\footnotetext{
${ }^{23} \mathrm{O}$ processo de treinamento pode ser supervisionado, em que o sistema aprende a associar determinados valores dos dados de entrada com rótulos fornecidos para seu treinamento; de reforço, quando as respostas do sistema são treinadas através do fornecimento de recompensas por respostas corretas; ou não supervisionado, em que o treinamento busca alinhar o sistema inteligente com uma métrica que reflete propriedades dos dados e não um resultado desejado (HAYKIN, 2008, p. 34-35).
}

${ }^{24}$ Parte da popularização deste paradigma da inteligência artificial nos últimos dados está ligada ao chamado big data, isto é, a formação de vastas bases de dados e as subsequentes possibilidades de extração de informação a partir destes dados. Sobre o tema, ver Gomes (2017).

${ }^{25}$ Sobre ambas as questões deste parágrafo, ver Burrell (2016). 
positivos de ambas as abordagens para a construção de sistemas mais adequados às peculiaridades das predições jurídicas, de natureza normativa.

\subsection{Sistemas baseados em conhecimento jurídico}

Sistemas baseados em conhecimento são sistemas inteligentes que realizam inferências a partir de representações internas de conhecimento (RUSSELL; NORVIG, 2010, p. 234). Essas representações do conhecimento são construídas de antemão, durante o projeto de um sistema computacional, mas, uma vez que elas sejam feitas, o sistema pode atualizar sua base de conhecimento a partir das informações que adquire do ambiente, aplicando as mesmas regras de inferência para lidar com situações percebidas.

No âmbito jurídico, esse raciocínio opera através da estrutura formal, que pode ser construída a partir de diferentes lógicas, como lógicas deônticas (HILPINEN; MCNAMARA, 2013), que lidam com conceitos como obrigações, proibições e permissões - seja por operadores modais, seja por meio de representações de conjuntos de normas, constitutivas, obrigatórias ou permissivas, como nas lógicas input/output - ou ainda lógicas de argumentação derrotável.

Lógicas de argumentação derrotável encontraram ampla aplicação em representação de conhecimento jurídico, a partir de duas abordagens principais: o raciocínio a partir de precedentes (case based reasoning) e o raciocínio a partir de regras (rule based reasoning). Essas lógicas modelam o raciocínio como inferências a partir de argumentos a favor ou contra determinada tese, incluindo informação sobre a força relativa desses argumentos. Um argumento pode ser uma estrutura inferencial complexa, que liga suas premissas a conclusões por meio de passos intermediários detalhados. ${ }^{26}$ Esses argumentos, por sua vez, podem ser atacados em diferentes junções e de diversas formas, e uma conclusão pode ser derivada se for possível construir um argumento a favor da conclusão que seja defensável contra todos os argumentos que o atacam.

Tal estrutura de argumentos, contra-argumentos, refutações e presunções alinha-se de forma direta com o raciocínio jurídico, tendo em vista que decisões judiciais são tomadas a partir da avaliação de argumentos das partes em oposição. Com isso, as lógicas de argumentação foram aplicadas com sucesso para representar vários aspectos do raciocínio jurídico. Entre as aplicações bem-sucedidas das

\footnotetext{
${ }^{26}$ De um ponto de vista técnico, tanto os passos intermediários quanto a conclusão final devem ser autorizados por operações de uma lógica dedutiva ou derrotável (não monotônica).
} 
lógicas de argumentação jurídica na inteligência artificial, temos modelos de raciocínio sobre argumentação oral em cortes (BENCH-CAPON; PRAKKEN, 2010), evidências processuais (BEX et al, 2003; VERHEIJ, 2016; entre outros) e raciocínio com precedentes judiciais (BENCH-CAPON, 2002; PRAKKEN; SARTOR, 1996; entre outros). Em particular, mais recentemente, modelos lógicos de argumentação foram desenvolvidos, nos quais ocorre o balanço de múltiplas considerações prós e contras - fatores do caso, razões, princípios e valores (HAGE, 2005; SARTOR, 2013; PRAKKEN et al., 2015; HORTY, 2011; entre outros). Além disso, alguns modelos formulam princípios racionais sobre o desenvolvimento dos precedentes no tempo e sobre a dinâmica de construção e alteração de conceitos em interpretações de leis e precedentes judiciais (entre os quais, MARANHÃO; SARTOR, 2019; HORTY; BENCH-CAPON, 2012).

Boa parte dos avanços de IA \& Direito sobre a interpretação de conceitos gira em torno da noção de "fator", que surgiu em dois programas de computador pioneiros em IA \& Direito: o sistema HYPO (ASHLEY, 1990) e o sistema CATO (ALEVEN, 2003). Fatores são abstrações ou estereótipos da descrição de um caso, que podem favorecer (fatores favoráveis) ou prejudicar (fatores contrários) uma conclusão legal. Por exemplo, o fator "gravidez resultante de estupro" é um fator favorável, no ordenamento jurídico brasileiro, à decisão de permitir ou não punir o aborto. Já o fator "procedimento não realizado por médico" é um fator que leva à proibição do aborto.

A atenção dada ao papel dos valores e propósitos levou a abordagens sobre a interpretação jurídica como um problema de decisão, ou seja, como uma escolha entre interpretações alternativas considerando a probabilidade daquela interpretação ou de decisões baseadas naquela interpretação ou, ainda, de consequências possíveis daquela interpretação em termos de promoção ou demoção dos valores relevantes. Naquelas abordagens, a escolha de uma interpretação é pautada nos efeitos positivos e negativos que as potenciais decisões podem ter em relação a valores ou objetivos que sejam tomados como relevantes, considerando as preferências relativas entre esses valores ou objetivos, e, em alguns modelos, também considerando a extensão na qual esses valores ou objetivos são alcançados ou impactados. Há também ligações entre essas abordagens e teorias da decisão qualitativas (KEENEY; RAIFFA, 1976), recentemente exploradas por Giovanni Sartor (2010; 2013).

Sistemas computacionais mais recentes, como o VJAP (GRABMAIR, 2017), desenvolvido para aplicação em temas de concorrência desleal, em particular sobre violação de segredo de indústria (trade secret law), procuram incorporar valores e 
propósitos perseguidos na construção de justificações para decisões judiciais. Com isso, o sistema faz predição do resultado do caso, ou seja, da provável decisão judicial, por meio de uma medida de confiança derivada dos grafos argumentativos, e gera textos com argumentos que justifiquem a predição fornecida.

Em todas essas abordagens, é possível reconstruir a lógica que o sistema utilizou para construir suas predições, o que fornece uma justificação racional para as decisões tomadas. Contudo, não só a construção desses sistemas exige o emprego de muito conhecimento específico sobre o tema, como também há a necessidade de se extrair manualmente a informação que os algoritmos usarão, o que exige, na prática, um trabalho substancial de pré-processamento para a construção e uso desses sistemas. Por isso, os sistemas baseados em conhecimento não são hoje tão aplicados quanto a sua sofisticação teórica poderia sugerir.

\subsection{Aplicações jurídicas do aprendizado de máquina}

Nos últimos anos, técnicas de aprendizado de máquina foram empregadas para prever resultados de decisões judiciais a partir de textos jurídicos. Um exemplo é o modelo construído por Nikolaos Aletras et al. (2016), que atingiu 79\% de acurácia ao avaliar se a Corte Europeia de Direitos Humanos decidiria pela existência de uma violação de direitos em um determinado caso. Em aplicações jurídicas, abordagens de Máquinas de Vetores-Suporte (SVM) têm obtido os melhores resultados, embora abordagens de deep learning venham ganhando espaço (CONTISSA et al., 2018).

De forma geral, esses modelos derivam suas predições de elementos textuais (features) que revelam padrões para as decisões. A construção de um modelo de aprendizado de máquina para o processamento de linguagem natural - o que inclui o processamento de textos jurídicos - envolve, em geral, quatro passos: (1) a compilação de um corpus de textos relevantes para o domínio da aplicação; (2) o préprocessamento dos textos desse corpus, para deixá-los em um formato que os algoritmos de processamento de linguagens naturais possam consumir; (3) a anotação destes textos, por meios automáticos ou manuais, para atribuir rótulos adequados (por exemplo, para dizer se um recurso foi provido); e (4) o treinamento do modelo que realizará as previsões desejadas (ECKART DE CASTILHO, 2018). Todas essas etapas podem se beneficiar do uso de conhecimentos específicos do domínio de aplicação como uma forma de melhorar o tratamento computacional dos textos analisados, como fazem sistemas como CLAUDETTE, desenvolvido na Universidade de Bologna, que detecta cláusulas abusivas em documentos que descrevem ou pedem concordância com as políticas de privacidade de sites (CONTISSA et al., 2018). 
Mesmo com o auxílio de conhecimento especialista, todavia, os padrões encontrados por um sistema inteligente muitas vezes são aqueles que um humano observaria ao desempenhar a mesma tarefa. Isso porque a predição normativa, típica do jurista, não é fundamentada na correlação empírica entre eventos, mas na apreciação dos fatores (características) do caso à luz das normas jurídicas aplicáveis, ou dos argumentos a favor ou contra determinada pretensão. Portanto, é difícil para o jurista humano entender quais são os eventos empíricos que o sistema julgou como relevantes e as correlações utilizadas que resultam no output do sistema.

Exemplo nesse sentido pode ser encontrado no trabalho de Verma et al. (2017), que desenvolveu um sistema com cerca de $75 \%$ de acurácia para predizer quando juízes das cortes de apelação norte-americanas divergiriam. Segundo a hipótese do trabalho, a divergência seria determinada por diferenças de natureza ideológica, porém os fatores mais correlacionados aos resultados foram: (i) a posição em que os juízes se sentavam no julgamento; (ii) o tamanho dos votos; e (iii) o número de citações de precedentes. Obviamente, na análise jurídica, fatores como esses são absolutamente irrelevantes. A predição jurídica de divergência liga-se à avaliação de convicções sobre teses jurídicas e princípios aceitos pelos juízes sob análise.

Assim, a pesquisa em inteligência artificial tem buscado o desenvolvimento de sistemas de inteligência artificial inteligíveis (Explainable Artificial Intelligence: xAI), tanto por meio da produção de sistemas capazes de explicar de forma mais simples o funcionamento de outros sistemas quanto pela construção de sistemas capazes de atingir bom desempenho a partir de mecanismos internos que incorporem representação de conhecimento jurídico.

\subsection{Inteligência artificial no Direito brasileiro}

Nos últimos anos, diversas empresas brasileiras, bem como empresas internacionais com atuação no mercado brasileiro, têm comercializado produtos tecnológicos voltados ao setor jurídico. ${ }^{27}$ De forma geral, essas empresas não costumam divulgar ao público as tecnologias que utilizam para suas soluções, mas pesquisas recentes (como TREVELIN et al., 2020) apontam para uma popularização do uso de técnicas baseadas em aprendizado de máquina, fator que é motivado ao menos em parte pela política de acesso aberto a dados judiciais.

\footnotetext{
${ }^{27} \mathrm{O}$ site da Associação Brasileira de Lawtechs e LegalTechs (AB2L, https://ab2l.org.br/radar-lawtechs/) revela que, em março de 2021, mais de 100 empresas do setor jurídico ofereciam produtos ou soluções voltadas ao público jurídico em sentido amplo. Embora nem todas essas empresas façam uso de inteligência artificial, algumas delas são dedicadas explicitamente a este tipo de aplicação, por exemplo como fornecedores de tecnologias inteligentes para o setor público ou como provedores de analytics (análise de dados) e jurimetria.
} 
Tendo em vista a importância do acesso a dados para o desenvolvimento do aprendizado de máquina, como descrito supra, o Conselho Nacional de Justiça (CNJ) estabeleceu, por meio da Resolução 334/2020, o Comitê Consultivo de Dados Abertos e Proteção de Dados no âmbito do Poder Judiciário. Este comitê tem por objetivo auxiliar o CNJ na construção de políticas de acesso a dados que equilibrem as demandas de transparência e desenvolvimento tecnológico, de um lado, e, de outro, a necessidade de proteção dos dados das pessoas eventualmente mencionadas no âmbito de documentos judiciais. Tais políticas não determinam o uso - ou não uso - de tecnologias em específico, mas, ao estabelecer padrões e medidas técnicas e administrativas para o adequado tratamento de dados judiciais, fornecem segurança jurídica para o desenvolvimento tecnológico no setor privado.

Em paralelo, o CNJ também atua no sentido de coordenar e amparar as diversas iniciativas de inteligência artificial desenvolvidas pelos próprios tribunais. Com base nos problemas identificados em cada corte e nas capacidades técnicas existentes em suas equipes de tecnologia, diversos tribunais desenvolveram sistemas internos para tarefas como a automação de tarefas identificadas como gargalos na operação dos tribunais, para a triagem de peças processuais, ou a sumarização de textos (SALOMÃO, 2020). Uma vez que tais iniciativas tiveram origem nos próprios tribunais, esses sistemas por vezes fazem uso de tecnologias e representações de dados distintas, o que dificulta o reaproveitamento de sistemas bem-sucedidos em outros contextos.

Diante desse obstáculo, que pode levar tribunais a despender esforços para resolver problemas já resolvidos alhures, a atuação recente do $\mathrm{CNJ}$ tem buscado fomentar a interoperabilidade e o compartilhamento do conhecimento entre os tribunais. Nesse sentido, destacam-se a adoção da Plataforma Sinapses (CONSELHO..., 2019), originada no Tribunal de Justiça de Rondônia e adotada na Portaria 271/2020 do CNJ como plataforma de inteligência artificial do Poder Judiciário. Com a adoção de uma plataforma comum, os tribunais poderão se beneficiar de uma infraestrutura técnica compartilhada, dedicando assim seus esforços tecnológicos à construção de modelos que tragam benefícios às operações dos tribunais.

\section{Perspectivas da inteligência artificial aplicada ao Direito}

\section{1 Abordagens híbridas e o problema da explicação das decisões}

Apesar de seus êxitos, ambos os paradigmas de inteligência artificial encontram limites à sua aplicação em questões jurídicas. No caso dos sistemas de conhecimento 
jurídico, o principal obstáculo consiste na dificuldade envolvida na codificação dos dados de um caso para que eles tenham uma forma que o sistema possa entender e processar, trabalho que hoje é majoritariamente feito por humanos. Para sanar essa dificuldade, há linhas prósperas de pesquisa voltadas ao desenvolvimento de sistemas para identificação automática de fatores juridicamente relevantes em textos jurídicos, com o emprego de modelos de espaços vetoriais (ASHLEY; FALAKMASIR, 2017), e de detecção automática e estruturação de argumentos (PALAU; MOENS, 2009; PATHAK; GOYAL; BHOMWICK, 2016). Embora essas tecnologias ainda precisem atingir maior grau de maturação, elas já contribuem para reduzir o trabalho humano necessário para a adoção de sistemas baseados em conhecimento jurídico.

Da mesma forma que o uso de técnicas de aprendizado de máquina pode servir para viabilizar o uso prático de sistemas baseados em conhecimento, as representações do domínio que esses proporcionam podem ser úteis para explicar como a inteligência artificial jurídica chega às suas conclusões. No campo do Direito, a IA inteligível é de particular importância, uma vez que qualquer ato ou decisão judicial ou administrativa somente é juridicamente válido na medida em que possa ser juridicamente justificado. ${ }^{28}$ Nesse domínio, o processo e o conteúdo de justificação são tão relevantes quanto o resultado. ${ }^{29}$

A necessidade de explicação não é apenas um requisito para que os sistemas inteligentes possam ser utilizados em aplicações jurídicas, mas é também um requisito para que as predições da inteligência artificial sejam eficazes. Posto que uma decisão judicial muitas vezes não é uma consequência imediata do "estímulo dos fatos" (ALETRAS et al., 2016) que possam ser detectados diretamente a partir do texto, uma predição de decisões judiciais não deve se limitar aos aspectos empíricos, mas também levar em conta as dinâmicas argumentativas que devem ser reconstruídas para que um sistema possa fazer uma predição adequada. Dessa forma, a incorporação de técnicas computacionais de tratamento da argumentação jurídica às técnicas já existentes de processamento de linguagem jurídica permitirá que os sistemas consigam identificar os argumentos presentes em um documento

\footnotetext{
${ }^{28}$ No ordenamento jurídico brasileiro, o Código de Processo Civil (artigo 489, II) impõe a apresentação da fundamentação como elemento essencial da sentença. Na Europa, a Corte Europeia de Direitos Humanos reconhece a presença de uma justificação como essencial para a possibilidade de recurso (Fomin v. Moldova (2011), No.36755/06, \$31) e para que as partes possam ter suas reclamações ouvidas, sendo assim parte das garantias de um processo justo (Suominen v. Finland (2003) No.37801/97, \$37). Sobre o papel das razões judiciais como mecanismos de controle de sistemas automatizados, ver Strandburg (2019).

${ }^{29}$ Como exemplo de projeto que busca fornecer explicações para decisões automatizadas no âmbito jurídico, temos o projeto NWO Forensic Science (https://www.ai.rug.nl/ verheij/nwofs/), coordenado por Bart Verheij, que busca desenvolver sistemas para gerar explicações sobre redes bayesianas e modelos probabilísticos de análise de evidências processuais, por meio de cenários e argumentos de forma que os modelos se tornem compreensíveis para juristas (VERHEIJ et al., 2016; VLEK, 2014).
} 
jurídico e, a partir disso, prever o resultado de uma forma ao mesmo tempo mais precisa e passível de explicação para os observadores humanos.

O caminho para a integração de modelos de predição é, de um lado, o desenvolvimento de programas capazes de identificar fatores relevantes ou argumentos em documentos jurídicos de tal modo que possam gerar premissas para sistemas computacionais que empreguem lógicas jurídicas e de argumentação. E, de outro, o desenvolvimento e refinamento dos sistemas de argumentação e construção de justificações com base em fatores e argumentos de modo a que possam processar argumentos e fatores extraídos automaticamente (por aprendizado de máquina) de textos e documentos jurídicos. Com isso, é possível desenvolver modelos de argumentação que possam, a partir de textos jurídicos com pouco ou nenhum tratamento especial, oferecer não só predições empíricas, obtidas a partir de correlações estatísticas, como também predições normativas, extraídas da força argumentativa do caso em relação a precedentes, bem como a construção de justificações jurídicas a partir de argumentos inteligíveis.

\subsection{Direito computável}

Outra linha de desenvolvimento da inteligência artificial jurídica consiste em tornar computáveis as normas existentes. Dada a ubiquidade dos sistemas de IA, passa a ser inviável a impossibilidade de fiscalização humana de todas as possíveis tomadas de decisão e ações por agentes digitais inteligentes, mesmo nos casos em que essas decisões são adequadamente explicadas. Então, torna-se imperativo que os sistemas inteligentes, em especial aqueles empregados nas aplicações juridicamente mais sensíveis, sejam capazes de ajustar suas ações em função das leis.

Uma primeira solução para esse problema consiste na adoção de técnicas de projeto para sistemas computacionais que assegurem que estes operem de forma compatível com a lei. É o caso de abordagens de proteção de dados by design ${ }^{30}$ e de técnicas por elas inspiradas, como contestability by design (ALMADA, 2019; BAYAMLIOĞLU, 2018; HILDEBRANDT, 2019) ou ethics by design (DIGNUM et al., 2018; ALMADA; ATTUX, 2018). Essas abordagens, em particular aquelas voltadas à proteção da privacidade, já são adotadas com variados graus de sucesso, mas enfrentam duas dificuldades principais: a possibilidade de conflitos entre os valores que essas abordagens tentam implementar (ALMADA; ATTUX, 2018; FEENBERG,

\footnotetext{
${ }^{30} \mathrm{O}$ artigo 25 do GDPR estabelece a necessidade de que sistemas adotem, por padrão e já desde o momento de sua concepção, medidas para a proteção de dados pessoais de seus usuários. No Brasil, este dispositivo encontra eco na LGPD (artigo 46, $\$ 2$ ). Para um panorama do debate acadêmico a respeito do tema, ver Almada, Maranhão e Sartor (no prelo).
} 
1992; VAN DE POEL, 2020); WARDMAN; LÖFSTEDT, 2018) e a falta de recursos conceituais e técnicos para antecipar problemas que podem ter impacto real (BREY, 2012; FLORIDI; STRAIT, 2020).

Em resposta a tais dificuldades, atualmente explora-se o desenvolvimento de sistemas inteligentes capazes de processar computacionalmente normas jurídicas ou morais. ${ }^{31}$ Como os sistemas de IA adaptam seu comportamento às circunstâncias, tal processamento não pode ser feito somente por programação regimental, mas exige a construção de representações formais das normas éticas ou jurídicas relevantes para um determinado domínio de aplicação. A partir destas representações, os sistemas inteligentes poderão raciocinar e aplicar regras éticas e legais no momento de escolha sobre seu curso de ação, considerando peculiaridades do contexto em vez de se limitar a determinações ex ante.

\section{Conclusão}

A difusão do uso de sistemas inteligentes tem o potencial de transformar a prática do Direito, não só por trazer novas questões a serem consideradas pelas profissões jurídicas, mas também pela automação de atividades jurídicas, começando por aquelas que envolvem trabalho repetitivo e posteriormente se sofisticando. Essas mudanças exigirão uma transformação no perfil do profissional jurídico, que precisará estar apto a lidar com o novo cenário social e com as novas tecnologias.

Em relação às aplicações da inteligência artificial ao Direito, esse processo de adaptação envolve tanto o uso das ferramentas de inteligência artificial pelos profissionais do Direito quanto o aproveitamento de seu conhecimento para a construção de sistemas computacionais que sejam capazes de realizar suas atividades de tratamento de dados de formas compatíveis com a lei. Portanto, a popularização da inteligência artificial exigirá profissionais capazes de lidar com as transformações tecnológicas e de operar em equipes interdisciplinares, que aproveitem as competências de juristas, cientistas da computação e outros profissionais para a construção de sistemas inteligentes que tenham efeitos positivos e protejam os direitos e interesses juridicamente tutelados em jogo.

\footnotetext{
${ }^{31}$ Ver, nesse sentido, o projeto europeu CompuLaw: https://cordis.europa.eu/project/id/833647. A partir de 2019, a Faculdade de Direito da Universidade de São Paulo passou a oferecer disciplinas de pós-graduação relativas ao direito computável.
} 


\section{Referências}

ABRUSIO, Juliana. Proteção de dados na cultura do algoritmo. São Paulo: Editora D’Plácido, 2020.

ALETRAS, Nikolaos et al. Predicting judicial decisions of the European Court of Human Rights: a Natural Language Processing perspective. PeerJ Computer Science, v. 2, e93, 2016.

ALEVEN, Vincent. Using background knowledge in case-based legal reasoning: a computational model and an intelligent learning environment. Artificial Intelligence, n. $150,2003$.

ALMADA, Marco. Human intervention in automated decision-making: toward the construction of contestable systems. In: INTERNATIONAL CONFERENCE ON ARTIFICIAL INTELLIGENCE AND LAW (ICAIL 2019), 17., 2019, Montreal. Proceedings [...]. Nova York: ACM Press, 2019.

ALMADA, Marco; ATTUX, Romis. Ethical design of social simulations. In: WORKSHOP SOBRE ASPECTOS SOCIAIS, HUMANOS E ECONÔMICOS DE SOFTWARE (WASHES 2018), 3. 2018, Porto Alegre, RS. Anais [...]. Porto Alegre: SBC, 2018. Disponível em: https:// sol.sbc.org.br/index.php/washes/issue/view/218. Acesso em: 20 abr. 2021.

ALMADA, Marco; MARANHÃO, Juliano; SARTOR, Giovanni. Article 25. Data protection by design and by default. In: SPIECKER GEN. DÖHMANN, Indra; PAPAKONSTANTINOU, Vagelis; HORNUNG, Gerrit; DE HERT, Paul (orgs.). European General Data Protection Regulation. Baden-Baden: Nomos, no prelo.

ASHLEY, Kevin. Modeling legal argument: reasoning with cases and hypotheticals. Cambridge: MIT Press, 1990.

ASHLEY, Kevin; FALAKMASIR, Mohammad Hassan. Utilizing vector space models for identifying legal factors from text. In: INTERNATIONAL CONFERENCE ON LEGAL KNOWLEDGE AND INFORMATION SYSTEMS - JURIX 2017, 30., 2017, Luxembourg. Proceedings [...]. Netherlands: IOS Press, 2017.

BAYAMLIOĞLU, Emre. Contesting automated decisions. European Data Protection Law Review (EDPL), v. 4, p. 433-446, 2018.

BAYAMLIOĞLU, Emre; LEENES, Ronald. The 'rule of law' implications of data-driven decision-making: a techno-regulatory perspective. Law, Innovation and Technology, v. 10, n. 2, p. 295-313, 2018.

BENCH-CAPON, Trevor J. M. The missing link revisited: the role of teleology in representing legal argument. Artificial Intelligence and Law, v. 10, p. 79-94, 2002.

BENCH-CAPON, Trevor J.M.; PRAKKEN, Henry. Using argument schemes for hypothetical reasoning in law. Artificial Intelligence and Law, v. 18, p. 153-174, 2010. 
BEX, Floris J. et al. Towards a formal account of reasoning about evidence: argumentation schemes and generalisations. Artificial Intelligence and Law, v. 11, p. 125-165, 2003.

BIETTI, Elettra. From ethics washing to ethics bashing: a view on tech ethics from within moral philosophy. In: CONFERENCE ON FAIRNESS, ACCOUNTABILITY, AND TRANSPARENCY - FAT ${ }^{*}$ 20, 2020, Barcelona. Proceedings [...]. New York: Association for Computing Machinery, 2020. p. 210-219.

BREY, Philip A. E. Anticipatory ethics for emerging technologies. NanoEthics, v. 6, n. 1, p. $1-13,2012$.

BRYSON, Joanna J.; THEODOROU, Andreas. How society can maintain human-centric artificial intelligence. In: TOIVONEN, Marja; SAARI, Eveliina (org.). Human-centered digitalization and services. Singapore: Springer Singapore, 2019. p. 305-323.

BURGESS, Peter et al. Towards a digital ethics. Bruxelas: Ethics Advisory Group, 2018.

BURRELL, Jenna. How the machine 'thinks': understanding opacity in machine learning algorithms. Big Data \& Society, v. 3, n. 1, p. 1-12, 2016.

CAPLAN, Robyn et al. Algorithmic accountability: a primer. Data \& Society, 2018. Disponível em: https://datasociety.net/output/algorithmic-accountability-a-primer/. Acesso em: 26 ago. 2018.

COMISSÃO EUROPEIA. COMUNICAÇÃO DA COMISSÃO AO PARLAMENTO EUROPEU, AO CONSELHO EUROPEU, AO CONSELHO, AO COMITÉ ECONÓMICO E SOCIAL E AO COMITÉ DAS REGIÕES. Plano Coordenado para a Inteligência Artificial. Disponível em: https://eur-lex.europa.eu/legal-content/PT/TXT/ HTML/?uri=CELEX:52018DC0795\&from=EN. Acesso em: 30 maio 2021. Número de referência COM: COM(2018)795 PT.

COMISSÃO EUROPEIA. Proposal for a Regulation on a European approach for Artificial Intelligence. Bruxelas: Comissão Europeia, 2021. Disponível em: https:// digital-strategy.ec.europa.eu/en/library/proposal-regulation-laying-down-harmonisedrules-artificial-intelligence. Acesso em: 30 maio 2021.

CONSELHO NACIONAL DE JUSTIÇA. Inteligência artificial no Poder Judiciário. Brasília, 2019. 40p. Disponível em: https://www.cnj.jus.br/wp-content/uploads/2020/05/Inteligencia_ artificial_no_poder_judiciario_brasileiro_2019-11-22.pdf. Acesso em: 20 abr. 2021.

CONTISSA, Giuseppe et al. CLAUDETTE meets GDPR: automating the evaluation of privacy policies using Artificial Intelligence. Brussels: European Consumer Organisation, (BEUC), 2018. Disponível em: https://www.beuc.eu/publications/beuc-x-2018-066_claudette_meets_ gdpr_report.pdf. Acesso em: 20 abr. 2021.

CORMEN, Thomas H. et al. Introduction to algorithms. 3. ed. Cambridge: The MIT Press, 2009. p. 5-6. 
DIGNUM, Virginia et al. Ethics by design: necessity or curse? In: THE 2018 AAAI/ACM CONFERENCE ON AI, ETHICS, AND SOCIETY, 2018, New Orleans. Proceedings [...]. New York: Association for Computer Machinery, 2018. p. 60-66.

DONEDA, Danilo Cesar Maganhoto et al. Considerações iniciais sobre inteligência artificial, ética e autonomia pessoal. Pensar - Revista de Ciências Jurídicas, v. 23, n. 4, p. 1-17, 2018.

ECKART DE CASTILHO, Richard. A legal perspective on training models for natural language processing. In: INTERNATIONAL CONFERENCE ON LANGUAGE RESOURCES AND EVAluATION, 11., 2018, Miyazaki, Japan. [Proceedings...]: LREC-2018). [s.l.], 2018.

FEENBERG, Andrew. Subversive rationalization: technology, power, and democracy. Inquiry, v. 35, n. 3-4, p. 301-322, 1992.

FLORIDI, Luciano; STRAIT, Andrew. Ethical foresight analysis: what it is and why it is needed? Minds and Machines, v. 30, n. 1, p. 77-97, 2020.

FLORIDI, Luciano, et al. AI4People: an ethical framework for a good AI society: opportunities, risks, principles, and recommendations. Minds and Machines, v. 28, 2018.

FRAZÃO, Ana; MULHOLLAND, Caitlin (org.). Inteligência artificial e Direito: ética, regulação e responsabilidade. 1. ed. São Paulo: Thomson Reuters Brasil, 2019.

FUX, Luiz; ÁVILA, Henrique; CABRAL, Trícia Navarro Xavier (org.). Tecnologia e Justiça multiportas: teoria e prática. Indaiatuba: Editora Foco, 2021.

GOMES, Rodrigo Dias de Pinho. Big data: desafios à tutela da pessoa humana na sociedade da informação. Rio de Janeiro: Lumen Juris, 2017.

GRABMAIR, Matthias. Predicting trade secret case outcomes using argument schemes and learned quantitative value effect tradeoffs. INTERNATIONAL CONFERENCE ON ARTIFICIAL INTELLIGENCE AND LAW (ICAIL 2017), 16., 2017, London. Proceedings [...].. New York: Association for Computing Machinery, 2017. p. 89-98.

HAGE, Jaap C. Comparing alternatives in the law. Artificial Intelligence and Law, v. 12, p. 181-225, 2005.

HAYKIN, Simon. Neural networks and learning machines. 3. ed. [New Jersey]: Prentice Hall, 2008. p. 34-35.

HILDEBRANDT, Mireille. Privacy as protection of the incomputable self: from agnostic to agonistic machine learning. Theoretical Inquiries in Law, v. 20, n. 1, 2019.

HILPINEN, Risto; MCNAMARA, Paul. Deontic Logic: a historical survey and introduction. In: GABBAY, Dov et al. (orgs.). Handbook of deontic logic and normative systems. London: College Publications, 2013. 
HORTY, John F. Rules and reasons in the theory of precedent. Legal Theory, v. 17, 2011.

HORTY, John F.; BENCH-CAPON, Trevor J. A factor-based definition of precedential constraint. Artificial Intelligence and Law, v. 20, p. 181-214, 2012.

JOBIN, Anna; IENCA, Marcello; VAYENA, Effy. The global landscape of AI ethics guidelines. Nature Machine Intelligence, v. 1, p. 389-399, 2019.

KAMINSKI, Margot. The right to explanation, explained. Berkeley Technology Law Journal, v. 34, n. 1, 2019.

KEENEY, Ralph L.; RAIFFA, Howard. Decisions with multiple objectives. New York: Wiley, 1976.

KOENE, Ansgar et al. A governance framework for algorithmic accountability and transparency. Brussels: European Union, 2019. Disponível em: https://www.europarl. europa.eu/RegData/etudes/STUD/2019/624262/EPRS_STU(2019)624262_EN.pdf.

Acesso em: 20 abr. 2021.

KUZNIACKI, Blazej. The artificial intelligence tax treaty assistant: decoding the principal purpose test. Bulletin for International Taxation, v. 72, n. 9, 2018.

LARSON, Christina. Who needs democracy when you have data? MIT Technology Review, 20 Aug. 2018. Disponível em: https://www.technologyreview.com/2018/08/20/240293/ who-needs-democracy-when-you-have-data/. Acesso em: 20 abr. 2021.

LEHR, David; OHM, Paul. Playing with the data: what legal scholars should learn about machine learning. University of California Davis Law Review, v. 51, p. 653-717, 2017.

MACHADO, Henrique Felix de Souza. Algoritmos, regulação e governança: uma revisão de literatura. Journal of Law and Regulation, v. 4, n. 1, p. 39-62, 2018.

MARANHÃO, Juliano; ALMADA, Marco. Inteligência artificial no setor de saúde: ética e proteção de dados. In: DALLARI, Analluza Bolivar; MONACO, Gustavo Ferraz de Campos (org.). LGPD na Saúde. 1. ed. São Paulo: Thomson Reuters Brasil, 2021.

MARANHÃO, Juliano; COUTINHO, Diogo R. Melhor investir do que regular. Correio Braziliense, 25 de março de 2019.

MARANHÃO, Juliano; SARTOR, Giovanni. Value assessment and revision in legal interpretation. In: INTERNATIONAL CONFERENCE ON ARTIFICIAL INTELLIGENCE AND LAW (ICAIL 2019), 17., 2019, Montreal. Proceedings [...]. New York: ACM Press, 2019.

MEDVEDEVA, Masha; VOLS, Michel; WIELING, Martijn. Using machine learning to predict decisions of the European Court of Human Rights. Artificial Intelligence and Law, v. 28, p. 237-266, 2020. Disponível em: https://link.springer.com/content/pdf/10.1007/s10506-01909255-y.pdf. Acesso em: 20 abr. 2021. 
MENDES, Laura Schertel; MATTIUZZO, Marcela. Discriminação algorítmica: conceito, fundamento legal e tipologia. Direito Público, v. 16, n. 90, p. 39-64, 2019. Disponível em: https://www.portaldeperiodicos.idp.edu.br/direitopublico/article/view/3766/Schertel\%20 Mendes\%3B\%20Mattiuzzo\%2C\%202019. Acesso em: 20 abr. 2021.

OLIVEIRA, Rafael Brito de. Utilização de ontologias para busca em base de dados de acórdãos do STF. 2017. Dissertação (Mestrado em Ciências da Computação) - Instituto de Matemática e Estatística, Universidade de São Paulo, São Paulo, 2017.

ONG, Rebecca. Recognition of the right to privacy on the Internet in China. International Data Privacy Law, v.1, n. 3, 2011.

PALAU, Raquel Mochales; MOENS, Marie-Francine. Argumentation mining: the detection, classification and structuring of arguments in text. In: INTERNATIONAL CONFERENCE ON ARTIFICIAL INTELLIGENCE AND LAW (ICAIL 2009), 12., 2009, Barcelona. Proceedings [...]. New York: Association for Computing Machinery, 2009. Disponível em: https://dl.acm. org/doi/proceedings/10.1145/1568234. Acesso em: 20 abr. 2021.

PASQUALE, Frank. The black box society: the secret algorithms that control money and information. Cambridge: Harvard University Press, 2015.

PATHAK, Arkanath; GOYAL, Pawan; BHOWMICK, Plaban. A two-phase approach towards identifying argument structure in Natural Language. In: WORKSHOP ON NATURAL LANGUAGE PROCESSING TECHNIQUES FOR EDUCATIONAL APLICATIONS, 3., 2016, Osaka. Proceedings of the NLPTEA 2016 Workshop. Osaka, 2016. Disponível em: https://www.aclweb.org/anthology/W16-49.pdf. Acesso em: 20 abr. 2021.

PRAKKEN, Henry; SARTOR, Giovanni. A dialectical model of assessing conflicting arguments in legal reasoning. Artificial Intelligence and Law, v. 4, p. 331-368, 1996.

PRAKKEN, Henry et al. A formalisation of argumentation schemes for legal case-based reasoning in ASPIC+. Journal of Logic and Computation, v. 25, n. 5, p. 1141-1166, 2015.

RUSSELL, Stuart J.; NORVIG, Peter. Artificial intelligence: a modern approach. 3. ed. Upper Saddle River: Prentice Hall, 2010. p. 234.

SALOMÃO, Luiz Felipe (coord.). Tecnologia aplicada à gestão dos conflitos no âmbito do Poder Judiciário brasileiro. Rio de Janeiro: Centro de Inovação, Administração e Pesquisa do Judiciário da Fundação Getúlio Vargas, 2020. Disponível em: https://ciapj.fgv.br/sites/ ciapj.fgv.br/files/estudos_e_pesquisas_ia_1afase.pdf. Acesso em: 20 abr. 2021.

SARRA, Claudio. Put dialectics into the machine: protection against automatic-decision-making through a deeper understanding of contestability by design. Global Jurist, v. 20, n. 3, 2020.

SARTOR, Giovanni. Fundamental legal concepts: A formal and teleological characterisation. Artificial Intelligence and Law, v. 21, pp. 101-142, 2010. 
SARTOR, Giovanni. The logic of proportionality: reasoning with non-numerical magnitudes. German Law Journal, v. 14, 2013.

SIMÕES-GOMES, Letícia; ROBERTO, Enrico; MENDONÇA, Jônatas. Viés algorítmico - um balanço provisório. Estudos de Sociologia, v. 25, n. 48, 2020.

SINGH, Jatinder et al.Responsibility and machine learning: part of a process. SSRN, 2016. p. 7. Disponível em: https://papers.ssrn.com/sol3/Delivery.cfm/SSRN_ID2860048_ code2330375.pdf?abstractid=2860048\&mirid=1. Acesso em: 20 abr. 2021 .

STRANDBURG, Katherine J. Rulemaking and inscrutable automated decision tools. Columbia Law Review, v. 119, n. 7, p. 1851-1886, 2019.

SWANDA, Gus. The Deficiencies of a Westphalian model for cyberspace: a case study of South Korean cyber security. International Journal of Korean Unification Studies, v. 25, n. 2, 2016.

TREVELIN, Bruna et al. Acesso a dados de processos judiciais no Brasil. São Paulo: Lawgorithm, 2020.

VAN DE POEL, Ibo. Core values and value conflicts in cybersecurity: beyond privacy versus security. In: CHRISTEN, Markus; GORDIJN, Bert; LOI, Michele (org.). The ethics of cybersecurity. Cham: Springer International Publishing, 2020. p. 45-71. (The International Library of Ethics, Law and Technology, 21)

VERHEIJ, Bart et al. Arguments, scenarios and probabilities: connections between three normative frameworks for evidential reasoning. Law, Probability \& Risk, v. 15, p. 35-70, 2016.

VERMA, S., PARTHASARATHY, A., CHEN, D. The genealogy of ideology: predicting agreements and persuasive memes in the U.S. Courts of Appeals. INTERNATIONAL CONFERENCE ON ARTIFICIAL INTELLIGENCE AND LAW (ICAIL 2017), 16., 2017, London. Proceedings [...].. New York: Association for Computing Machinery, 2017.

VLEK, Charlotte S. Building bayesian networks for legal evidence with narratives: a case study evaluation. Artificial Intelligence and Law, v. 22, n. 4, p. 375-421, 2014.

WAGNER, Ben. Ethics as an escape from regulation: from ethics-washing to ethics-shopping? In: HILDEBRANDT, Mireille (ed.). Being profiled: cogitas ergo sum. Amsterdam: Amsterdam University Press, 2018.

WARDMAN, Jamie K.; LÖFSTEDT, Ragnar. Anticipating or accommodating to public concern? risk amplification and the politics of precaution reexamined. Risk Analysis, v. 38, n. 9, p. 1802-1819, 2018. 


\section{Legislação citada}

BRASIL. Lei no 13.105, de 16 de março de 2015. Código de Processo Civil. Brasília: Presidência da República, [2021]. Disponível em: https://www.planalto.gov.br/ccivil_03/_Ato20152018/2015/Lei/L13105.htm\#art1046. Acesso em: 20 abr. 2021.

BRASIL. Lei no 13.709, de 14 de agosto de 2018. Lei Geral de Proteção de Dados Pessoais (LGPD). Brasília: Presidência da República, [2019]. Disponível em: https://www.planalto. gov.br/ccivil_03/_ato2015-2018/2018/lei/113709.htm. Acesso em: 20 abr. 2021.

CONSElHO NACIONAL DE JUSTIÇA. Portaria no 271, de 4 de dezembro de 2020. Regulamenta o uso de Inteligência Artificial no âmbito do Poder Judiciário. Disponível em: https://atos.cnj.jus.br/files/original234208202012155fd949d04d990.pdf. Acesso em: 20 abr. 2021.

CONSELHO NACIONAL DE JUSTIÇA. Resolução no 332, de 21 de agosto de 2020. Dispõe sobre a ética, a transparência e a governança na produção e no uso de Inteligência Artificial no Poder Judiciário e dá outras providências. Disponível em: https://atos.cnj. jus.br/files/original191707202008255f4563b35f8e8.pdf. Acesso em: 20 abr. 2021.

CONSELHO NACIONAL DE JUSTIÇA. Resolução no 334, de 21 de setembro de 2020. Institui o Comitê Consultivo de Dados Abertos e Proteção de Dados no âmbito do Poder Judiciário. Disponível em: https://atos.cnj.jus.br/files/original193417202009225f6a51b9a757c. pdf. Acesso em: 20 abr. 2021.

UNIÃO EUROPEIA. Regulamento(UE) 2016/679 do Parlamento Europeu e do Conselho, de 27 de abril de 2016. Relativo à proteção das pessoas singulares no que diz respeito ao tratamento de dados pessoais e à livre circulação desses dados e que revoga a Diretiva 95/46/ CE (regulamento Geral sobre a Proteção de Dados). [2018]. Disponível em: https://eur-lex. europa.eu/legal-content/PT/TXT/PDF/?uri=CELEX:02016R0679-20160504\&from=EN.

Acesso em: 20 abr. 2021. 